\title{
PREDICTABILITY OF DRUG SENSITIVITY IN MULTI-SPECIES ENVIRONMENTS
}

\author{
CARLOS REDING ${ }^{1 *}$ \\ ${ }^{1}$ Department of Biosciences, University of Exeter, EX4 4QD, United Kingdom. \\ Current address: Department of Genetics, School of Medicine, Stanford University. Stanford, CA 94304. \\ *Corresponding author: reding@stanford. edu
}

Increasing evidence suggests that microbial pathogens and tumours can have multiple sensitivities to the same drug, but the underlying mechanism is still unknown. Here I show that competition for a shared resource, like nutrients from the blood, can change the drug content of a focal genotype and therefore alter its drug response with respect to controlled laboratory assays. A simple mathematical model illustrates that neighbouring genotypes can act as carbon or drug sink depending on their drug sensitivity. Thus, concentrating or diluting the drug contained in competing genotypes. Aided by this model, I halved the minimum inhibitory concentration for tetracycline of Escherichia coli MC4100 in $24 \mathrm{~h}$ sensitivity tests. The effect was maintained after 80 generations-in bacterial timescalesfollowing serial passages akin to those used in evolutionary biology. It is hypothesised that microbes could be used to fight cancer given the regressions reported when bacteria grow in their microenvironment. My model provides a rationale to deploy, or indeed design, specific microbial competitors that curb drug sensitivity of cancer and microbial pathogens.

\section{INTRODUCTION}

The notion of cultures where a genotype is isolated and grown in purified form is fundamental to design antimicrobial $(1-3)$ and anticancer $(4,5)$ therapies. Once purified, clinicians can expose the genotypetumour $(4,5)$ or microbe $(6)$ - to a range of drug concentrations and measure its sensitivity using metrics such as the minimum inhibitory concentration (6) or half-maximal inhibitory concentration (5). But there is a problem: Therapies do not target these genotypes in isolation, but in multi-species environments $(7,8)$. Reproducing in vivo the inhibition observed in laboratory assays is challenging given the diversity pathogens and tumours thrive in. Antibiotic therapies, for example, can fail in polymicrobial environments (9). Similarly, the efficacy of anticancer drugs such as fluoropyrimidines is highly variable due to the host microbiota (10) during colorectal cancer treatments. So, here I asked the following: What is the inhibitory concentration in dynamic, living environments?

Below I show a simple mathematical model suggesting that neighbouring genotypes act as carbon or drug sink when they engage in competition for shared resources with a focal genotype (microbe or tumour). This results in the concentration or dilution of drug contained within competing genotypes and, therefore, change their drug response. Interestingly, the change in sensitivity is predictable, and to demonstrate this I used the model to reduce the minimum inhibitory concentration of tetracycline in Escherichia coli. Perhaps more importantly, the bacterium remained hyper-sensitive to this drug for more than 80 generations, resembling the conjectured outcome of evolution-proof therapies (II). The model can be used to predict the likelihood of drug-tolerance of a pathogen or tumour based on phenotypic properties of their neighbouring genotypes, such as growth rate or drug sensitivity. 


\section{RESUlTS}

Drug response of a focal species is determined by susceptibility of its neighbouring species. Consider $j$ phenotypically distinct species competing for a limited resource, $C$, and exposed to a drug, $A$, supplied at concentration $A_{e}(0)=A_{0}$, cast as the following model:

$$
\begin{aligned}
& \dot{S}_{j}=\overbrace{G_{j}(C) S_{j}}^{\text {Growth }} \cdot \overbrace{I_{j}(A)}^{\text {Inhibition }}, \\
& \dot{A}_{j}=\overbrace{-d A_{j}}^{\text {Decay }}+\overbrace{\varphi_{j}\left(A_{e}-A_{j}\right) S_{j}}^{\text {Fick's Diffusion }},
\end{aligned}
$$

Here, $\dot{S}_{j}$ and $\dot{A}_{j}$ represent the density of individuals per unit volume from species $j$ and their content of drug $A$ over time, respectively, with initial conditions $S_{j}(0)=S_{j 0}$ and $A_{j}(0)=0 . U_{j}(C)$, the uptake rate of resource $C$-supplied at concentration $C_{0}$ —of individuals from species $j$, is a saturating Monod function proportional to the maximal uptake rate,

$$
U_{j}(C):=\bar{\mu}_{j} \frac{C}{K_{j}+C}
$$

where $K_{j}$ is the half-saturation parameter and the affinity of individuals from species $j$ for the limited resource $C$ is given by $1 / K_{j}$. Their growth rate (i.e. absolute fitness) at a given resource concentration is denoted by $G_{j}(C):=U_{j}(C) \cdot y_{j}$, where $y_{j}$ is the biomass yield per unit of resource in individuals from species $j$. Their growth inhibition, by $\operatorname{drug} A$, is described qualitatively by the inhibition function (12)

$$
I_{j}(A):=\frac{1}{1+\left(A_{j} / \kappa_{j}\right)^{\alpha}}, \text { where } 0 \leq I_{j}(A) \leq 1 .
$$

This function is dimensionless and has two parameters. First, the Hill coefficient $\alpha$ which characterises the cooperativity of the inhibition. And second, $\kappa_{j}$ is the affinity of drug $A$ for its target and it can be derived from the drug concentration required to halve the maximal growth rate, so that $A_{50}=1 / \kappa_{j}$ (12). For the sake of simplicity, I assumed that drug $A$ diffuses from the environment into cells of species $j$, and vice versa, with a diffusion coefficient $\varphi_{j}$; and part of $A$ being lost to chemical stability (13) at a rate $d$. Indeed, antimicrobial and anticancer drugs can diffuse into cells passively or facilitated by membrane proteins (14-16). However, they can also be actively uptaken by membrane transporters (14, 15).

For my first computation I set the number of species $j=2$, to facilitate later experimental validation, where $I_{1}(A)=I_{2}(A)$ and $G_{1}(C)=G_{2}(C)$. Thus, individuals from both species are sensitive to $A$ and phenotypically identical. Given Equation 3, the density of individuals from either species as pure cultures, after $24 \mathrm{~h}$ of incubation, declines with higher drug concentrations consistently with standard clinical protocols $(6,17)$ (Figure $1 \mathrm{~A})$. To allow experimental validation, I calculated the concentration 

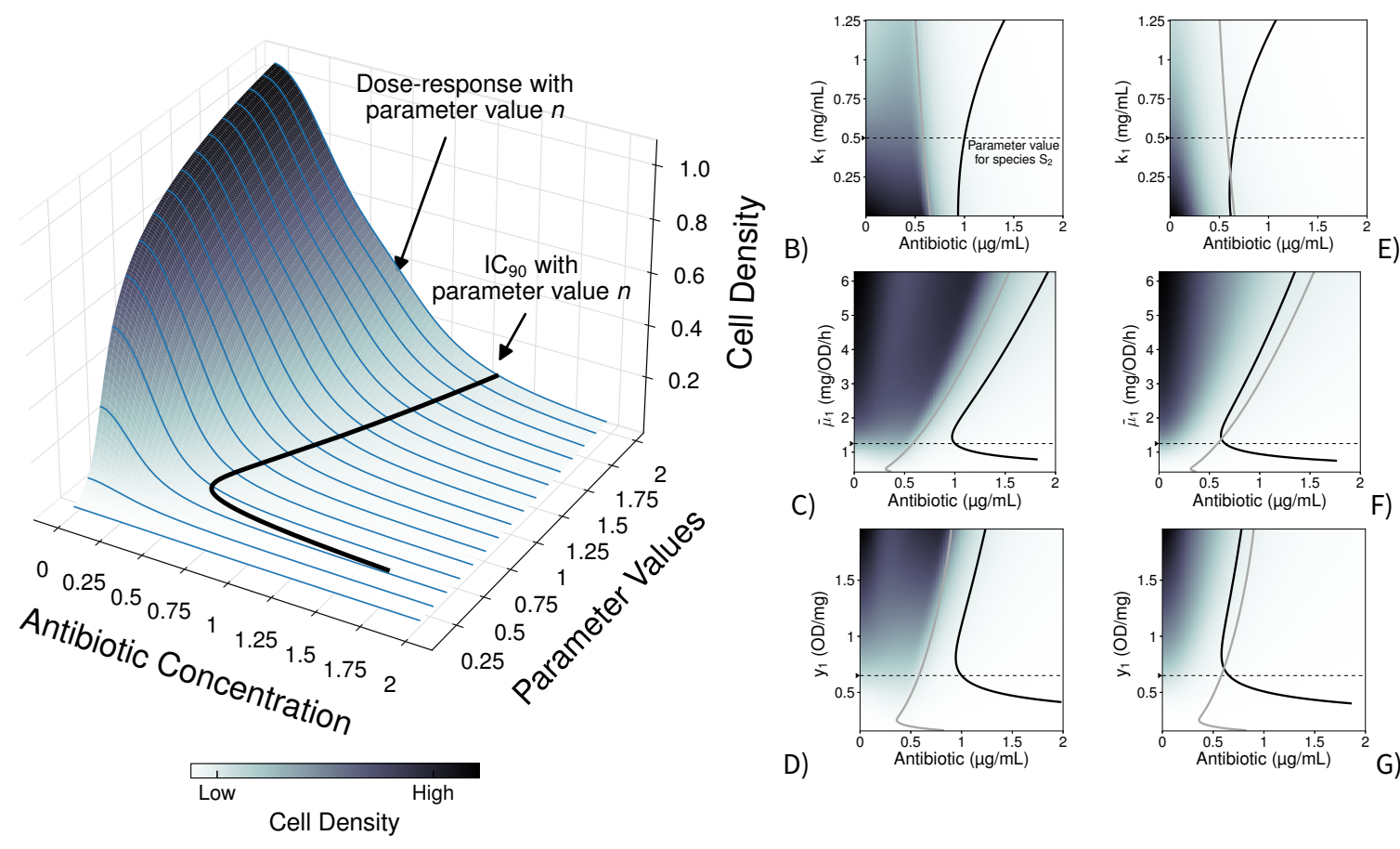

E)

C)

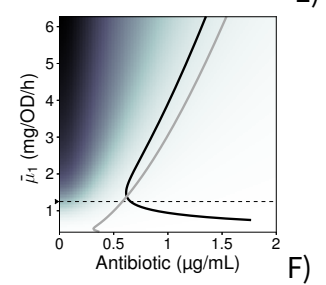

D)
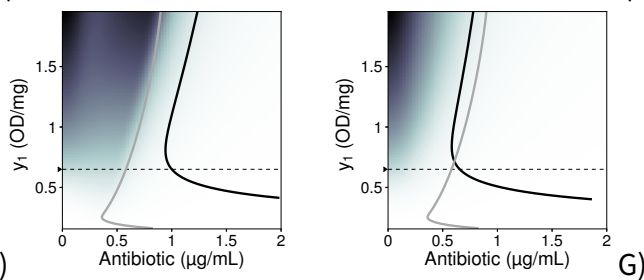

Figure 1. $S_{\mathbf{1}}$ drug sensitivity profiles in pure and mixed culture growth conditions alongside competing species $\boldsymbol{S}_{\mathbf{2}}$.

A) Growth of species $S_{1}$, with different parameter values $\left(k_{1}, \bar{\mu}_{1}\right.$, and $\left.y_{1}\right)$, after $24 \mathrm{~h}$ of growth in the presence of different antibiotic concentrations. I aggregated the resulting dose-response profiles (blue) to create a density map from low predicted cell density (white) to high predicted cell density (black). B-D) IC 90 , antibiotic concentration inhibiting $90 \%$ $\left(\mathrm{IC}_{90}\right)$ the growth predicted without drug, resulting with different parameters values for the half-saturation parameter $k_{1}(\mathrm{~B})$, maximal carbon up-take $\bar{\mu}_{1}(\mathrm{C})$, or biomass yield $y_{s}(\mathrm{D})$ in equation 1 when species $\mathrm{S}_{2}$ is drug-sensitive. The IC $\mathrm{C}_{90}$ for species $S_{1}$ growing as pure cultures is shown in grey, and growing in mixed culture with $S_{2}$ are shown in black. The parameter values for species $\mathrm{S}_{2}$ were fixed at a value noted by a black arrow on the $y$-axis, followed by a dotted black line. E-G) Change in $\mathrm{IC}_{90}$, as in Figures $\mathrm{B}-\mathrm{C}$ ), when the competing species $\mathrm{S}_{2}$ is not drug-sensitive (resistant or tolerant).

of $A$ inhibiting the growth of the pure cultures by $90 \%\left(\mathrm{IC}_{90}\right)$ as commonly used in clinic laboratories $(18-20)$. The drug sensitivity of each species depends on the values for the parameters $K, \bar{\mu}$, and $y$ of Equation 2 (Figure $1 B-D$, grey), with values that increase the density of individuals resulting in higher $\mathrm{IC}_{90}$. This is consistent with the inoculum effect (21), whereby sensitivity tests using larger inocula also report higher minimum inhibitory concentrations. However, the relationship between the parameters $\bar{\mu}$ and $y$ and the resulting $\mathrm{IC}_{90}$ is non-monotone, meaning certain values for these parameters maximise susceptibility for drug $A$.

This phenomenon is exacerbated if both species grow in mixed culture conditions, where both become phenotypically more tolerant to drug $A$ (Figure $1 \mathrm{~B}-\mathrm{D}$, black). If I were to target, say, individuals from species $S_{1}$, doing so when the species is surrounded by $S_{2}$ would require more drug. This is the case of pancreatic ductal adenocarcinoma treated with gemcitabine when can bacteria grow in its microenvironment (22). More generally, genotypes analog to $S_{1}$ should increase their drug tolerance when they are surrounded by similarly sensitive species.

To test this hypothesis, I mixed equal proportions of Escherichia coli Wyl and Salmonella typhimurium 
SL1344 in minimal media supplemented with different concentrations of tetracycline (see Methods). This antibiotic can diffuse passively into cells of both gram-negative species (23), who also have similar sensitivity to this antibiotic, $0.232 \pm 0.003$ and $0.276 \pm 0.016 \mu \mathrm{g} / \mathrm{mL}$ of tetracycline (mean $\mathrm{IC}_{90} \pm 95 \%$ confidence, with $n=8$ replicates, see Methods). This approximates to $I_{1}(A) \approx I_{2}(A)$, as laid out by the theory above. The chromosome of $E$. coli Wyl carries $y f p$, gene encoding a yellow fluorescence protein (YFP), so I tracked its density in mixed culture conditions. Consistently with Equations $1 \mathrm{a}-\mathrm{d}$, the bacterium was around $23 \%$ more tolerant to tetracycline when it grew in mixed culture with $S$. typhimurium (Mann-Whitney U-test $p=1.554 \times 10^{-4}$, ranksum $=36$ with $n=8$ replicates, Figure $2 \mathrm{~A}$ ).

Next, I explored in the model the case where individuals from both species have different sensitivities to drug $A\left(I_{1}(A) \neq I_{2}(A)\right.$ ). This scenario is akin to pathogens such as $C$. difficile growing alongside human cells $(24)$ where the latter are unaffected by the drug $\left(I_{2}(A) \approx 1\right)$. The model now predicts a subset of values for $K, y$, and $\bar{\mu}$ that make $S_{1}$ more sensitive to the drug in the presence of individuals from species $S_{2}$ (Figure $1 \mathrm{E}-\mathrm{G}$ ). To test this prediction, I mixed equal proportions of two constructs of Escherichia coli with different sensitivities to tetracycline. One construct is Wyl, used above, who is sensitive to the antibiotic. The other construct is $\mathrm{GB}(\mathrm{c})$, harbouring a non-transmissible plasmid carrying the gene tet(36) (25) and, therefore, resistant to the drug. Tetracycline binds to the bacterial ribosome, inhibiting protein synthesis (26), and tet(36) provides ribosomal protection against tetracycline (25) without degrading the antibiotic. The $\mathrm{IC}_{90}$ for this construct was $6.106 \pm 0.272 \mu \mathrm{g} / \mathrm{mL}$ of tetracycline (mean $\mathrm{IC}_{90} \pm 90 \%$ confidence with $n=8$ replicates). Now, $I_{1}(A) \ll I_{2}(A)$ satisfies the assumption above. The $\mathrm{IC}_{90}$ for $E$. coli Wyl was $0.232 \pm 0.003 \mu \mathrm{g} / \mathrm{mL}$ of tetracycline as pure culture. Growing alongside drug-resistant $\mathrm{GB}(\mathrm{c})$, however, it was $0.112 \pm 0.003 \mu \mathrm{g} / \mathrm{mL}$ (Figure $2 \mathrm{~B}$ ).

Neighbouring species $\mathbf{S}_{\mathbf{2}}$ determines drug availability for $\mathbf{S}_{\mathbf{1}}$. Above I noted that parameter values leading to higher density of individuals in pure culture, also led to higher $\mathrm{IC}_{90}$. When $I_{1}(A) \approx I_{2}(A)$, Equations $1 \mathrm{a}-\mathrm{d}$ suggest that individuals from one species change the drug availability, measured as relative drug molecules per individual, for the other. Thus, when species $S_{2}$ captures through diffusion its share of drug in mixed culture conditions, there is less of it available for species $S_{1}$ resulting in less drug per individual (Figure SiA-C) - and vice versa. However, when $I_{1}(A) \neq I_{2}(A)$, by virtue of different affinities of drug $A$ for each species $\left(\kappa_{1} \neq \kappa_{2}\right)$, the drug diffuses more rapidly from the least sensitive species back into the environment-re-exposing its sensitive counterpart. Thus, the change in drug content in the most sensitive species occurs through a different mechanism. The least sensitive species, given its higher tolerance to antimicrobial $A$, can maintain its growth and therefore it can remove a higher share of the limited resource, $C$. Consequently, the growth of the most sensitive species is limited (27), leaving more drug per individual of this species (Figure S1D-F). This competition for a shared resource is akin to that found, for example, between Salmonella typhimurium biofilms and cancer cells, where they compete for access to nutrients in the bloodstream-resulting in the bacteria inhibiting cancer growth (28).

To verify this rationale, I estimated the content of tetracycline in E. coli Wyl by dividing the bacterium's culture density, measured in relative fluorescence units to allow tracking in mixed culture conditions, by the concentration of tetracycline defining its $\mathrm{IC}_{90}$. The estimates resemble closely the theoretical predictions in Figure 2C: E. coli Wyl contains approximately $20 \%$ less tetracycline growing next to Salmonella typhimurium (Figure 2D) and 65\% more tetracycline growing alongside drug-resistant $\mathrm{GB}(\mathrm{c})$ (Figures 2E). 
A)

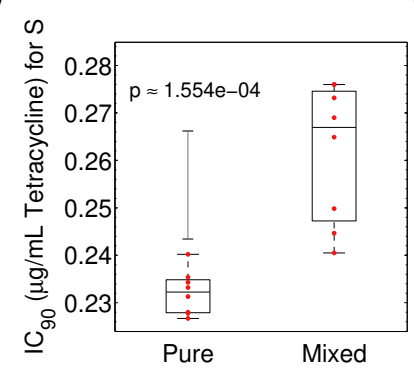

B)

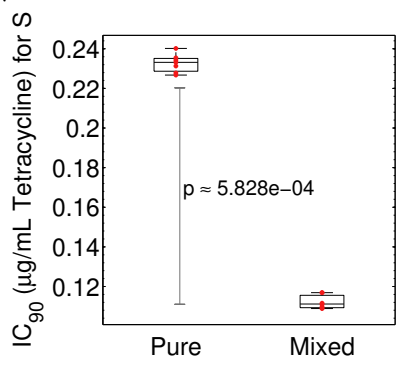

C)

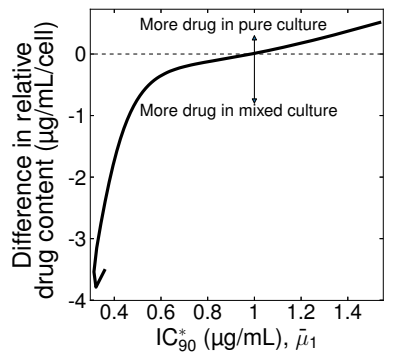

D)

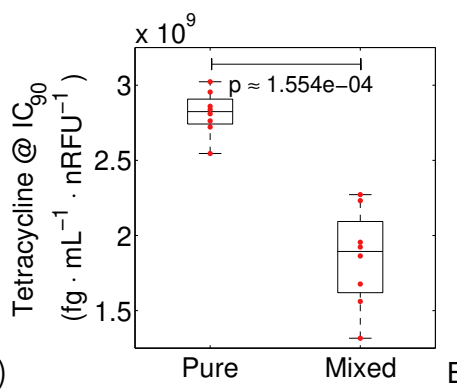

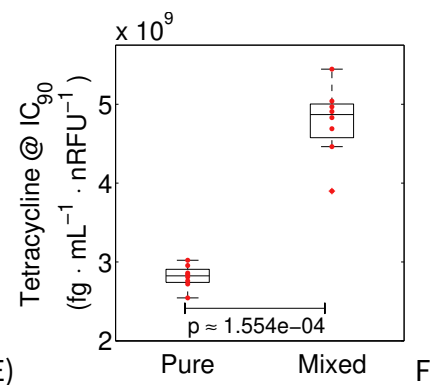

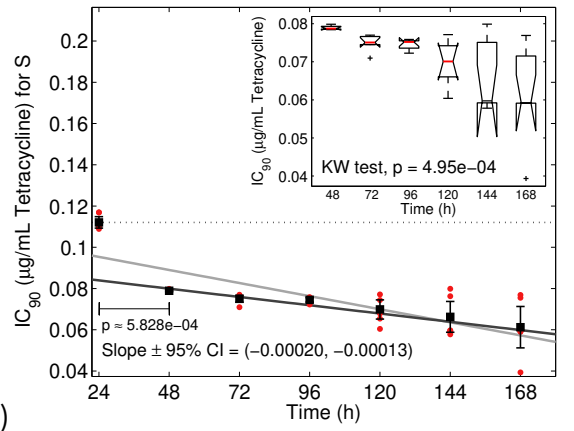

Figure 2. Changes in $I_{90}$ of drug-sensitive Escherichia coli Wyl are consistent with theoretical predictions. A-B) $\mathrm{IC}_{90}$ for tetracycline of Escherichia coli Wyl in pure culture, and in mixed culture with Salmonella typhimurium (A) and Escherichia coli GB(c) (B). The IC $\mathrm{C}_{90}$ for S. typhimurium in pure culture was $0.276 \pm 0.016 \mathrm{tg} / \mathrm{mLof}$ tet racycline (mean \pm $95 \%$ confidence), and $6.106 \pm 0.272 \mu \mathrm{g} / \mathrm{mL}$ for $E$. coli $\mathrm{GB}(\mathrm{c})$. The box plot shows the median (centre of the box), 25th, and 75th percentile of the dataset. The whiskers extend to the most extreme data points that are not outliers, which are individually represented. Raw data is represented as red dots. The $p$ value shown corresponds to a Mann-Whitney U-test. C) Theoretical difference in relative drug content-antibiotic molecules per cell-of $\mathrm{S}_{1}$ between pure culture conditions, and mixed culture with drug-sensitive $S_{2}$ for different $\bar{\mu}$ values (for all parameters in Figure S1). Positive values denote higher content of antibiotic per cell in pure culture conditions, whereas negative values denote higher antibiotic per cell in mixed culture. Lack of difference is represented by a horizontal, dotted line. D-E) Estimation of tetracycline content from experimental data of E. coli Wyl growing alongside Salmonella typhimurium (D) and E. coli $\mathrm{GB}(\mathrm{C})$ (E). The box plots show the median (centre of the box), 25th, and 75th percentile of the dataset. The whiskers extend to the most extreme data points that are not outliers, which are individually represented. Raw data is represented as red dots. The $p$ value shown corresponds to a Mann-Whitney U-test. F) Variation in $\mathrm{IC}_{90}$ of $E$. coli Wyl in mixed culture over time. The errorbars denote mean $\mathrm{IC}_{90}$ and $95 \%$ confidence, and raw data is shown as red dots. The $p$ value shown corresponds to a MannWhitney U-test. I fitted a linear model to $I C_{90}$ data including (grey) and excluding the $\mathrm{IC}_{90}$ at $24 \mathrm{~h}$, and showed the slope parameter of the case with the lowest $p$. The inset show the $p$ value of a Kruskal Wallis one-way ANOVA applied to IC 90 data excluding that measured at $24 \mathrm{~h}$. The box plot shows the median in red, 25th, and 75th percentile of the dataset. The whiskers extend to the most extreme data points that are not outliers, which are individually represented as a black cross.

Now, experiments of parallel evolution show that acr, operon responsible for the multi-drug efflux pump AcrAB-TolC (29), undergoes genomic amplification in E. coli MC4100 (30). Consequently, MC4100 overcomes the exposure to doxycycline, a type of tetracycline drug, within five days given its increased capacity to remove antibiotic molecules (30). Other strains of E. coli show identical adaptation (31). Interestingly, many types of cancer acquire resistance to chemotherapy through multidrug-efflux pumps (32). Note that resistance through efflux pumps does not alter the predictions presented in the manuscript (see Supplementary Text). To test whether Wyl, MC410o derivative sensitive to tetracycline, overcomes its exposure to the drug I propagated a culture containing equal proportions of $E$. coli Wyl 
and $\mathrm{GB}$ (c) for $168 \mathrm{~h}$. If Wyl acquires a mutation, such as the amplification of acr, that protects it against tetracycline I would expect greater $\mathrm{IC}_{90}$ over time. However, as Figure $2 \mathrm{~F}$ illustrates, the $\mathrm{IC}_{90}$ of Wyl was further reduced during this period.

\section{Discussion}

Martins etal. (33) showed that cell competition can act as a tumour suppressor mechanism, but what are the parameter of such competitions? One of the main challenges that cancer therapies face is the lack of reproducibility due to either culture protocols (5) or interactions with the host microbiota (10). Bacteria, on the other hand, lack this drawback while showing striking similarities with tumours: Drugs diffuse through similar membrane proteins (14), and their response to antibiotics can be mediated by multi-drug efflux pumps (29) or neighbouring cells $(34,35)$. In the case of Escherichia coli, population densities are highest in metabolically inefficient cells (36), showing a behaviour redolent of the Warburg effect commonly described in cancers (37). Jacques Monod wrote (38) "what is true of E. coli must be true of elephants" and, phenotypically, it certainly looks close enough to cancers in the context of cell competition and response to drugs.

While the drug sensitivity of tumours and bacteria can be measured in controlled laboratory assays, their response in vivo depends on neighbouring cells (33-35). The parameters of this interaction, and the mechanism, is not well understood, and so, it is not possible to predict what the sensitivity will be in in vivo. If emerging evidence suggests that bacteria could be used to fight cancer $(28,39)$, there needs to be a framework that can be used to predict qualitatively drug response in vivo given certain assumptions. I believe my model provides the first such framework to understand tumour-bacteria, or tumour-cell interactions and a rationale to deploy—or indeed design — microbial competitors to predictably curb a tumour's sensitivity. Despite the simplicity of the model, its predictions are consistent with prior in vivo datasets. For example, Geller et al. (22) showed that pancreatic ductal adenocarcinoma cells contained less gemcitabine when bacteria grew in the tumour's microenvironment, causing tumour cells to have higher tolerance to the drug. The authors could not report a mechanism to explain this observation, but my model suggests one: These bacteria acted as drug sink, reducing the exposure to gemcitabine of the tumour.

\section{METHODS}

Media and Strains. The strains of Escherichia coli GB(c) and Wyl were a gift from Remy Chait and Roy Kishony, and Salmonella typhimurium SL1344 a gift from Markus Arnoldini and Martin Ackermann. Experiments were conducted in M9 minimal media supplemented with $0.4 \%$ glucose (Fisher Scientific \#G/0500/53) and $0.1 \%$ casamino acids (Duchefa \#C1301.0250), supplemented with tetracycline. M9 minimum media (50X) was made by mixing equal proportions ( $\mathrm{vol} / \mathrm{vol}$ ) of two parts, part $\mathrm{A}$ and part $\mathrm{B}$, and diluted accordingly to $1 \mathrm{X}$. Part A (50X) contains $350 \mathrm{~g} / \mathrm{L}$ of $\mathrm{K}_{2} \mathrm{HPO}_{4}$ (Sigma \#P3786) and 100g/L of $\mathrm{KH}_{2} \mathrm{HPO}_{4}$ (Sigma \#P9791); whereas part B (50X) contains $29.4 \mathrm{~g} / \mathrm{L}$ of trisodium citrate (Sigma \#S1804), $50 \mathrm{~g} / \mathrm{L}$ of $\left(\mathrm{NH}_{4}\right)_{2} \mathrm{SO}_{4}$ (Sigma \#A4418), and $10.45 \mathrm{~g} / \mathrm{L}$ of $\mathrm{MgSO}_{4}$ (Fisher Scientific \#M/1050/53). I made tetracycline stock solutions from powder stock (Duchefa \#0150.0025) at $5 \mathrm{mg} / \mathrm{mL}$ in deionised water. Subsequent dilutions were made from this stock and kept at $4^{\circ} \mathrm{C}$. 
Acknowledgements: The author thanks Robert Beardmore for laboratory support. with $0-0.5 \mu \mathrm{g} / \mathrm{mL}$ of tetracycline (for E. coli Wyl and S. typhimurium) or $0-15 \mu \mathrm{g} / \mathrm{mL}$ (for E. coli GB(c)), with an overnight of each strain to measure drug sensitivity in pure cultures. For sensitivity assays of Wyl in mixed culture conditions I inoculated the microtitre plate, containing $150 \mu \mathrm{g} / \mathrm{mL}$ of media supplemented with $0-0.5 \mu \mathrm{g} / \mathrm{mL}$ of tetracycline, with equal proportions of two overnight cultures: Wyl + $\mathrm{GB}(\mathrm{c})$ or Wyl + S. typhimurium and using 8 technical replicates for each drug concentration.

I incubated the microtitre plate at $30^{\circ} \mathrm{C}$ in a commercial spectrophotometer and measured the optical density of each well at $600 \mathrm{~nm}\left(\mathrm{OD}_{600}\right)$, yellow florescence for Wyl (YFP excitation at $505 \mathrm{~nm}$, emission at $540 \mathrm{~nm}$ ), and cyan fluorescence for GB(c) (CFP at $430 \mathrm{~nm} / 480 \mathrm{~nm}$ ) every $20 \mathrm{~min}$ for $24 \mathrm{~h}$. I defined the minimum inhibitory concentration as the tetracycline concentration able to inhibit $90 \%$ of the growth observed in the absence of antibiotic after the $24 \mathrm{~h}$ incubation period.

Culture readings. Fluorescence protein genes were constitutively expressed with an approximately constant fluorescence to optical density ratio (Figure S2). The number of colony forming units (CFU) is positively correlated with optical density measured at $600 \mathrm{~nm}\left(O D_{600}\right)$ (Figure S3). Thus, I normalised fluorescence readings with respect to optical density readings, using the ratio optical density to fluorescence that I in pure culture conditions, to track the relative abundance of Wyl in mixed culture conditions. Time series data set were blank corrected prior to calculating the minimum inhibitory concentration.

Evolutionary dataset. I grew a mixed culture containing equal proportions (cell/cell) of each species in a 96-well microtitre plate containing $150 \mu \mathrm{g} / \mathrm{mL}$ of $\mathrm{M} 9$ media supplemented with $0-0.5 \mu \mathrm{g} / \mathrm{mL}$ of tetracycline. After $24 \mathrm{~h}$, I propagated the mixed culture into a new microtitre plate containing fresh media and antibiotic. Growth data was blank corrected and used to calculate the $\mathrm{IC}_{90}$ after every passage.

Code availability: A non-parameterized, python3 implementation of equations $1 \mathrm{a}-\mathrm{d}$ can be found at https://github.com/rc-reding/papers/tree/master/EvolProof_2020. Table I contains a list of parameter values used.

Table 1. Model parameters for Equations $1 a-d, 2$ and 3.

\begin{tabular}{cll}
\hline Parameter & Description & Value \\
\hline $\bar{\mu}_{j}$ & Maximal carbon uptake rate & $1.25 \mathrm{mg} / \mathrm{OD} / \mathrm{h}$ \\
$K_{j}$ & Half-saturation constant & $0.5 \mathrm{mg} / \mathrm{mL}$ \\
$y_{j}$ & Biomass yield & $0.65 \mathrm{OD} / \mathrm{mg}$ \\
$d$ & Drug degradation rate & $10^{-4} / \mathrm{h}$ \\
$\kappa_{j}$ & Affinity of drug A for species type $j$ & $0.1 \mathrm{~mL} / \mu g$ \\
$\varphi_{j}$ & Diffusion coefficient & $0.1 \mathrm{~mL} / \mathrm{OD} / \mathrm{h}$ \\
$A_{0}$ & Initial drug concentration & $2 \mu \mathrm{g} / \mathrm{mL}$ \\
$C_{0}$ & Initial carbon concentration & $2 \mathrm{mg} / \mathrm{mL}$ \\
\hline
\end{tabular}

Sensitivity assay. I inoculated a $96-$ well microtitre plate, containing $150 \mu \mathrm{g} / \mathrm{mL}$ of media supplemented 
Competing interests: The author declares no competing interests.

\section{REFERENCES}

1. Russell, B. M., Udomsangpetch, R., Rieckmann, K. H., et al. Simple in vitro assay for determining the sensitivity of Plasmodium vivax isolates from fresh human blood to antimalarials in areas where P. vivax is endemic. Antimicrob. Agents. Chemother. 47, 170-173 (2003).

2. Reinthaler, F., Posch, J, Feierl, G, et al. Antibiotic resistance of E. coli in sewage and sludge. Water Res. 37, 1685-1690 (2003).

3. Bennett, J., Dolin, R. \& Blaser, M. Principles and Practice of Infectious Diseases Churchill Livingstone v. 1. ISBN: 9781455748013 (Elsevier - Health Sciences Division, 2014).

4. Niepel, M., Hafner, M., Chung, M. \& Sorger, P. K. Measuring cancer drug sensitivity and resistance in cultured cells. Curr. Protoc. Chem. Biol. 9, 55-74 (2017).

5. Larsson, P., Engqvist, H., Biermann, J., et al. Optimization of cell viability assays to improve replicability and reproducibility of cancer drug sensitivity screens. Sci. Rep. 10, 1-12 (2020).

6. Performance Standards for Antimicrobial Susceptibility Testing; Twenty-Second Informational Supplement M100-S22 3. Clinical and Laboratory Standards Institute (2012). ISBN: 1-56238-786-3.

7. Peters, B. M., Jabra-Rizk, M. A., Graeme, A., Costerton, J. W. \& Shirtliff, M. E. Polymicrobial interactions: impact on pathogenesis and human disease. Clin. Microbiol. Rev. 25, 193-213 (2012).

8. Chen, J., Domingue, J. C. \& Sears, C. L. Microbiota dysbiosis in select human cancers: evidence of association and causality in Semin. Immunol. 32 (2017), 25-34.

9. Simón-Soro, A. \& Mira, A. Solving the etiology of dental caries. Trends Microbiol. 23, 76-82 (2015).

10. Scott, T. A., Quintaneiro, L. M., Norvaisas, P., et al. Host-microbe co-metabolism dictates cancer drug efficacy in C. elegans. Cell 169, 442-456 (2017).

11. Bell, G. \& MacLean, C. The Search for 'Evolution-Proof' Antibiotics. Trends Microbiol. (2017).

12. Klumpp, S., Zhang, Z. \& Hwa, T. Growth rate-dependent global effects on gene expression in bacteria. Cell 139, 1366-1375 (2009).

13. Gómez-Pacheco, C., Sánchez-Polo, M, Rivera-Utrilla, J \& López-Peñalver, J. Tetracycline degradation in aqueous phase by ultraviolet radiation. Chem. Eng. J. 187, 89-95 (2012).

14. Braun, V., Bös, C., Braun, M. \& Killmann, H. Outer membrane channels and active transporters for the uptake of antibiotics. The J. Infect. Dis. 183, S12-S16 (2001).

15. Nikolaou, M., Pavlopoulou, A., Georgakilas, A. G. \& Kyrodimos, E. The challenge of drug resistance in cancer treatment: a current overview. Clin. Exp. Metastasis 35, 309-318 (2018).

16. Muley, H., Fadó, R., Rodríguez-Rodríguez, R. \& Casals, N. Drug uptake-based chemoresistance in breast cancer treatment. Biochem. Pharmacol., 113959 (2020).

17. Antimicrobials susceptibility testing version 5.0 European Committee on Antimicrobial Susceptibility Testing (European Committee on Antimicrobial Susceptibility Testing, Jan. 2015).

18. Cedillo-Rivera, R \& Munoz, O. In-vitro susceptibility of Giardia lamblia to albendazole, mebendazole and other chemotherapeutic agents. J. Med. Microbiol. 37, 221-224 (1992). 
19. Cottrell, M. L., Hadzic, T. \& Kashuba, A. D. Clinical pharmacokinetic, pharmacodynamic and drug-interaction profile of the integrase inhibitor dolutegravir. Clin. Pharmacokinet. 52, 981-994 (2013).

20. Baumgartner, M., Bayer, F., Pfrunder-Cardozo, K. R., Buckling, A. \& Hall, A. R. Resident microbial communities inhibit growth and antibiotic-resistance evolution of Escherichia coli in human gut microbiome samples. PLoS Biol. 18, e3000465 (2020).

21. Eng, R., Smith, S. \& Cherubin, C. Inoculum effect of new beta-lactam antibiotics on Pseudomonas aeruginosa. Antimicrob. Agents. Chemother. 26, 42-47 (1984).

22. Geller, L. T., Barzily-Rokni, M., Danino, T., et al. Potential role of intratumor bacteria in mediating tumor resistance to the chemotherapeutic drug gemcitabine. Science 357, 1156-1160 (2017).

23. Grossman, T. H. Tetracycline antibiotics and resistance. Cold Spring Harb. Perspect. Med. 6, a025387 (2016).

24. Burke, K. E. \& Lamont, J. T. Fecal Transplantation for Recurrent Clostridium difficile Infection in Older Adults: A Review. J. Am. Geriatr. Soc. 61, 1394-1398 (2013).

25. Whittle, G, Whitehead, T. R., Hamburger, N, Shoemaker, N. B., Cotta, M. A., et al. Identification of a new ribosomal protection type of tetracycline resistance gene, tet(36), from swine manure pits. Appl. Environ. Microbiol. 64, 4151-4158 (2003).

26. Epe, B \& Woolley, P. The binding of 6-demethylchlortetracycline to $70 S, 50 S$ and $30 S$ ribosomal particles: a quantitative study by fluorescence anisotropy. EMBO J. 3, 121-126 (1984).

27. Day, T., Huijben, S. \& Read, A. F. Is selection relevant in the evolutionary emergence of drug resistance? Trends Microbiol. 23, 126-133 (2015).

28. Duong, M. T.-Q., Qin, Y., You, S.-H. \& Min, J.-J. Bacteria-cancer interactions: bacteria-based cancer therapy. Exp. Mol. Med. 51, 1-15 (2019).

29. Okusu, H., Ma, D. \& Nikaido, H. AcrAB efflux pump plays a major role in the antibiotic resistance phenotype of Escherichia coli multiple-antibiotic-resistance (Mar) mutants. J. Bacteriol. 178, 306308 (1996).

30. Pena-Miller, R., Laehnemann, D., Jansen, G., et al. When the most potent combination of antibiotics selects for the greatest bacterial load: the smile-frown transition. PLoS Biol. 11, e1001540 (2013).

31. Ayari, Jessica, P., Fabio, G., et al. Using a Sequential Regimen to Eliminate Bacteria at Sublethal Antibiotic Dosages. PLoS Biol. 13, e1002104 (Apr. 2015).

32. Eckford, P. D. \& Sharom, F. J. ABC efflux pump-based resistance to chemotherapy drugs. Chem. Rev. 109, 2989-3011 (2009).

33. Martins, V. C., Busch, K., Juraeva, D., et al. Cell competition is a tumour suppressor mechanism in the thymus. Nature 509, 465-470 (2014).

34. Lasa, I. \& Solano, C. Polymicrobial infections: Do bacteria behave differently depending on their neighbours? Virulence 9, 895-897 (2018).

35. Galera-Laporta, L. \& Garcia-Ojalvo, J. Antithetic population response to antibiotics in a polybacterial community. Sci. Adv. 6, eaaz5108 (2020). 
bioRxiv preprint doi: https://doi.org/10.1101/2020.12.14.422780; this version posted April 12, 2021. The copyright holder for this preprint (which was not certified by peer review) is the author/funder, who has granted bioRxiv a license to display the preprint in perpetuity. It is made available under aCC-BY-NC 4.0 International license.

10

36. Reding-Roman, C., Hewlett, M., Duxbury, S., et al. The unconstrained evolution of fast and efficient antibiotic-resistant bacterial genomes. Nat. Ecol. Evol. 1, 0050 (2017).

37. Koppenol, W. H., Bounds, P. L. \& Dang, C. V. Otto Warburg's contributions to current concepts of cancer metabolism. Nat. Rev. Cancer 11, 325-337 (2011).

38. Struhl, K. From E. coli to elephants. Nature 417, 22-23 (2002).

39. Sedighi, M., Zahedi Bialvaei, A., Hamblin, M. R., et al. Therapeutic bacteria to combat cancer; current advances, challenges, and opportunities. Cancer Med. 8, 3167-3181 (2019). 


\section{SUPPLEMENTARY TEXT}

Sensitivity modulation by species $\mathbf{S}_{\mathbf{2}}$ is independent of resistance mechanism. In the main text, I defined whether species $S_{2}$ is sensitive to drug $A$ based on the numerical value of $\kappa_{j}$ in equation 3-which denotes the affinity of drug $A$ for its target. This is equivalent to mutations, say, in the bacterial ribosome that reduce the affinity of ribosome-binding antibiotics for their target (1). But other resistance mechanisms such, as efflux pumps, do not act on the binding affinity of a drug for its target. Efflux pumps can have broad specificity (2), thus, able to protect against multiple drugs simultaneously $(3,4)$. To explore whether the predictions in the main text are robust to different mutation types, I redefined equations $\mathrm{ib}$ and $\mathrm{c}$ to accommodate efflux pumps as resistance mechanism (5):

$$
\dot{S}_{j}=\overbrace{G_{j}(C) S_{j}}^{\text {Growth }} \cdot \overbrace{I_{j}(A)}^{\text {Inhibition }},
$$

(1)

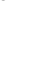

0

1

$$
\dot{A}_{j}=\overbrace{-d A_{j}}^{\text {Decay }}+\overbrace{\left(\varphi_{j}\left(A_{e}-A_{j}\right)-\frac{v^{*} p_{j}}{k_{m}^{*}+p_{j}}\right) S_{j}}^{\text {Fick's Diffusion with Efflux }}
$$

$$
\dot{A}_{e}=-d A_{e}-\sum_{j=1}^{i}\left(\varphi_{j}\left(A_{e}-A_{j}\right)-\frac{v^{*} p_{j}}{k_{m}^{*}+p_{j}}\right) S_{j}
$$

$$
\dot{C}=-\sum_{j=1}^{i} \overbrace{U_{j}(C) S_{j}}^{\text {C-Uptake }}
$$

Here $v^{*}$ represents the maximal efflux rate; $k_{m}^{*}$ the half-saturation constant associated where the affinity of the pump for its substrate, $A$, is given by $1 / k_{m}^{*}$; and $0 \leq p_{j} \leq 1$ is the expression level of $j-1$ copies of the efflux pump gene based on the limited abundance of DNA polymerase transcription complex (6). The abundance of this pump depends on the number of genes $j-1$ encoding efflux pump. The parameter $p_{j}$ is monotonically increasing and bounded in $j$, controlled by a dimensionless constant $\gamma$ in the Michaelis-Menten function $p_{j}=(j-1) /(1+\gamma(j-1))$ and $p_{j} /\left(k_{m}^{*}+p_{j}\right)$ the probability that a given drug molecule is bound to the pump. Thus, species $S_{1}$ does not express any efflux pump (no copies, as $j-1=0$ ) whereas species $S_{2}$ does indeed express the efflux pump. The remaining parameters are described in the main text with $\kappa_{1}=\kappa_{2}$.

As Figure $\mathrm{S}_{4}$ illustrates, efflux pumps do not change the effect that competing genotypes have on species $\mathrm{S}_{1}$. However, this resistance mechanism does increase even further the relative abundance of $\operatorname{drug} A$ in species $S_{1}$. Drug $A$ also diffuses into competing species $S_{2}$ until it reaches equilibrium, and will diffuse back into the environment as the abundance of $A$ declines, effectively re-exposing $\mathrm{S}_{1}$. Active efflux exacerbates this effect by actively moving $A$ molecules from within $S_{2}$ back into the environment. The result, shown in Figures $\mathrm{S} 4 \mathrm{~A}-\mathrm{C}$, is further inhibition of $\mathrm{S}_{1}$ with respect to the mechanism used in the main text, as noted by its lower $\mathrm{IC}_{90}$. 


\section{SUPPLEMENTARY REFERENCES}

1. Hu, M., Nandi, S., Davies, C. \& Nicholas, R. A. High-level chromosomally mediated tetracycline resistance in Neisseria gonorrhoeae results from a point mutation in the rpsJ gene encoding ribosomal protein S1o in combination with the mtrR and penB resistance determinants. Antimicrobial agents and chemotherapy 49, 4327-4334 (2005).

2. Murakami, S., Nakashima, R., Yamashita, E. \& Yamaguchi, A. Crystal structure of bacterial multidrug efflux transporter AcrB. Nature 419, 587-593 (2002).

3. Eckford, P. D. \& Sharom, F. J. ABC efflux pump-based resistance to chemotherapy drugs. Chem. Rev. 109, 2989-3011 (2009).

4. Blair, J. M., Bavro, V. N., Ricci, V., et al. AcrB drug-binding pocket substitution confers clinically relevant resistance and altered substrate specificity. Proc. Natl. Acad. Sci. U.S.A. 112, 35113516 (2015).

5. Ayari, Jessica, P., Fabio, G., et al. Using a Sequential Regimen to Eliminate Bacteria at Sublethal Antibiotic Dosages. PLoS Biol. 13, e1002104 (Apr. 2015).

6. Nierlich, D., Rutter, W. \& Fox, C. Molecular Mechanisms in the Control of Gene Expression Fig. 3 in p.139. ISBN: 9781483273938 (Elsevier Science, 2013).

\section{SUPPLEMENTARY Figures}

A)

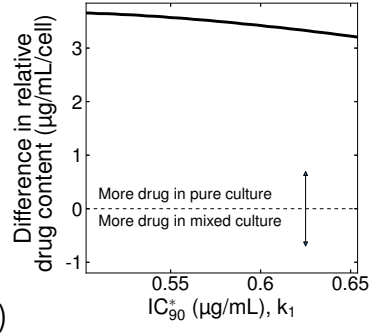

D)

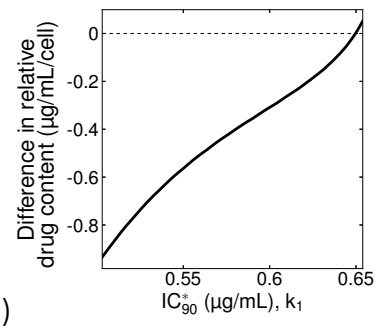

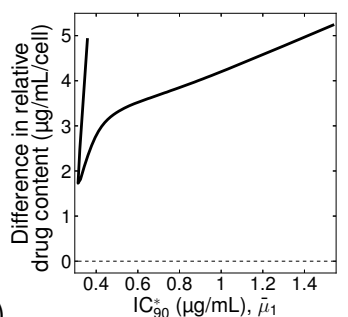

B)

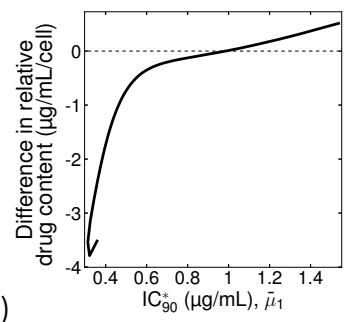

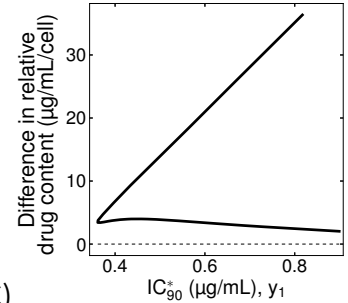

C)

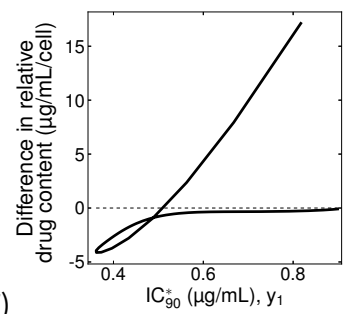

Figure S1. Drug concent in individuals from species $S_{\mathbf{1}}$ in pure and mixed growth conditions. A-C) Theoretical difference in relative drug content-antibiotic molecules per cell-of $S_{1}$ between pure culture conditions, and mixed culture with drug-sensitive $S_{2}$. A), B) and C) illustrate the prediction when changing the parameter $k, \bar{\mu}$, and $y$, respectively. The difference is positive $(>0)$ when the relative content of antibiotic is higher in pure culture conditions, whereas is negative $(<0)$ when the content is higher in mixed culture conditions. Lack of difference is represented by a horizontal, dotted line. D-F) Theoretical difference in relative drug content-antibiotic molecules per cell-of $S_{1}$ between pure culture conditions, and mixed culture with drug-insensitive $S_{2}$. A), B) and C) illustrate the prediction when changing the parameter $k, \bar{\mu}$, and $y$, respectively. The difference is positive $(>0)$ when the relative content of antibiotic is higher in pure culture conditions, whereas is negative $(<0)$ when the content is higher in mixed culture conditions. Lack of difference is represented by a horizontal, dotted line. 


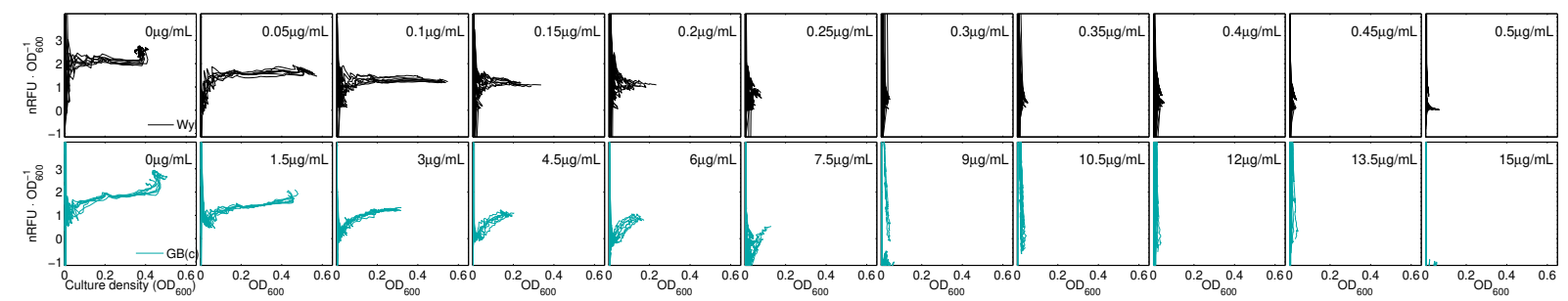

Figure S2. Changes in relative fluorescence over time in both Wyl and GB(c) strains. Raw change in florescence, per optical density units, measured every $20 \mathrm{~min}$ for $24 \mathrm{~h}$ for E. coli Wyl (black) and GB(c). Each column represents the data

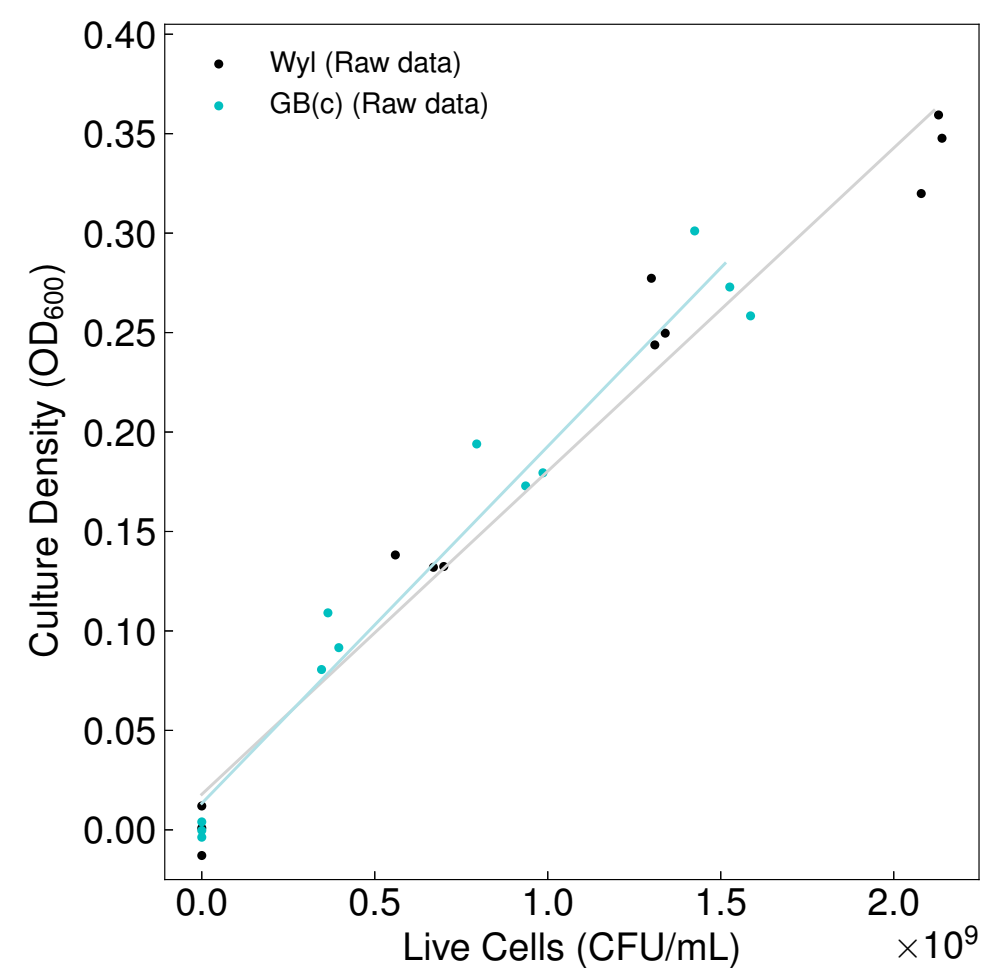

Figure S3. Calibration curve to translate optical density data to number of Escherichia coli cells. I fitted the linear model $a=b x+c$ to optical density and colony counting data (dots) to calculate the number of optical density units $\left(\mathrm{OD}_{600}\right)$ per cell. $a$ denotes the optical density readings measured at $600 \mathrm{~nm}, c$ the crossing point with the $y$-axis when $x=0$, and $b$ the conversion factor between optical density and number of cells $(x)$. I interpolating optical density readings to calculate the number of cells within a culture as $x=(a-c) / b$. For the strain $\mathrm{S}, b=1.62 \times 10^{-10} \mathrm{OD} \cdot \mathrm{mL}$. $C F U^{-1}$ and $c=1.78 \times 10^{-2} O D$, whereas for $\mathrm{R} b=1.79 \times 10^{-10} O D \cdot m L \cdot C F U^{-1}$ and $c=1.33 \times 10^{-2} O D$. 
bioRxiv preprint doi: https://doi.org/10.1101/2020.12.14.422780; this version posted April 12, 2021. The copyright holder for this preprint (which was not certified by peer review) is the author/funder, who has granted bioRxiv a license to display the preprint in perpetuity. It is made available under aCC-BY-NC 4.0 International license.
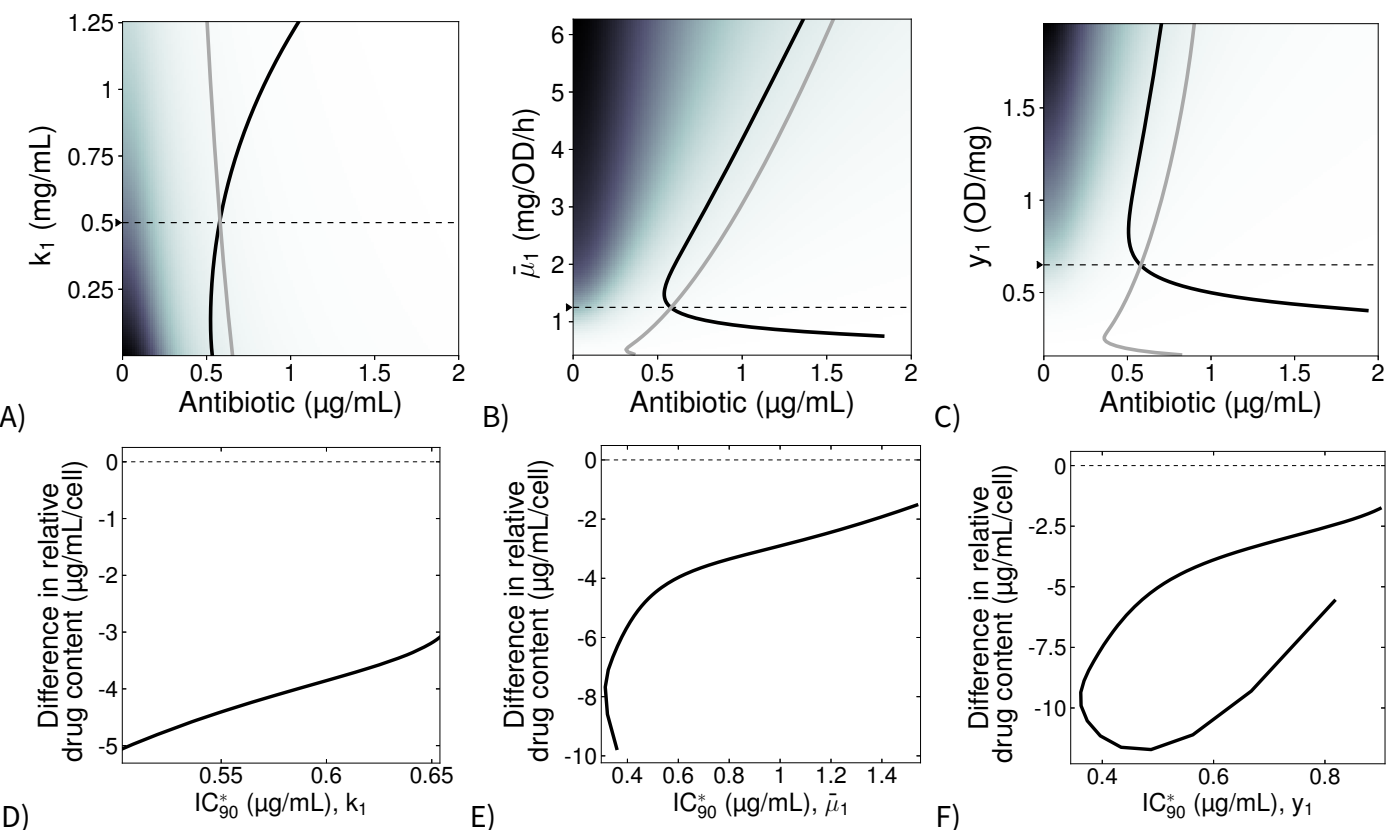

Figure S4. Drug concent in individuals from species $S_{1}$ in pure and mixed growth conditions when competing genotypes avoid drug-inhibition through efflux pumps. A-C) $I C_{90}$, antibiotic concentration inhibiting $90 \%\left(I C_{90}\right)$ the growth predicted without drug, resulting with different parameters values for the half-saturation parameter $k_{1}(\mathrm{~B})$, maximal carbon up-take $\bar{\mu}_{1}(\mathrm{C})$, or biomass yield $y_{s}$ (D) in equation 1 when species $S_{2}$ is drug-resistant through efflux pumps. The $I C_{90}$ for species $S_{1}$ growing as pure cultures is shown in grey, and growing in mixed culture with $S_{2}$ are shown in black. The parameter values for species $S_{2}$ were fixed at a value noted by a black arrow on the $y$-axis, followed by a dotted black line. D-F) Theoretical difference in relative drug content-antibiotic molecules per cell-of $S_{1}$ between pure culture conditions, and mixed culture with drug-insensitive $S_{2}$. D), E) and F) illustrate the prediction when changing the parameter $k, \bar{\mu}$, and $y$, respectively. The difference is positive $(>0)$ when the relative content of antibiotic is higher in pure culture conditions, whereas is negative $(<0)$ when the content is higher in mixed culture conditions. Lack of difference is represented by a horizontal, dotted line. 